\title{
An Experimental Investigation of the Vibration Characteristics of a Piping System
}

\author{
E.M.A. Rabeih ${ }^{\dagger}$ \\ Mechanical Design Department, Faculty of Engineering-Mataria, P.O. 11718, Helwan University, Cairo, Egypt
}

(Received 23 May 2000; accepted 18 September 2000)

\begin{abstract}
In this paper, an experimental investigation of the vibration characteristics of a piping system is presented. A PVC simple plane water-fully-filled piping system has been considered. Preliminary experiments have been carried out to obtain the dynamic modulus of elasticity of the pipe material using vibration measurements. The main experimental work has been carried out to obtain the resonance frequencies of the piping system and corresponding modal damping ratios from a set of frequency response functions (FRF) of the piping structure and fluid pressure measurements. The experimental results for the resonance frequencies have been compared with the theoretical values that were obtained using a finite element technique. The experimental results have been shown to be in good agreement with the theoretical values. The effect of the fluid on the modal damping ratios has been investigated by comparing the modal damping ratios of the empty piping system with the fully-filled one.
\end{abstract}

${ }^{\dagger}$ Member of the International Institute of Acoustics and Vibration (IIAV)

\section{INTRODUCTION}

The vibration study of pipes conveying fluid has gained considerable attention in the second half of the current century. A great deal of work has been done on various aspects of the dynamic behaviour of pipes conveying fluid. An extensive literature review on this subject can be found in reference ${ }^{1}$. The vibration that occurs in fluid-filled piping systems is of interest in a variety of industrial applications. In addition to the serious problems due to the vibration of piping structures, the fluid pressure can display important fluctuations. Even weak pressure fluctuations may be amplified to significant values if they coincide with the frequencies of a fluctuating pressure source such as found in reciprocating pumps. This oscillating pressure exerts forces on the piping system at discontinuities of the system such as, elbows, tees, valves, etc... These excitation forces may seriously increase the fatigue loads on the pipe and other components of the piping system. On the other hand, the dynamic behaviour of the fluid and the piping structure affect each other. This means that the propagating pressure of the fluid in a rigid pipe differs from the fluid pressure in an elastic pipe. Also the piping structure can be excited in different modes by the pressure variations in the contained fluid. Many authors, e.g. ${ }^{2-4}$, have identified the importance of the fluid-structure interaction in piping systems and its effect on the propagating fluid pressure and the vibrational behaviour of the piping structure. However, most of them have presented theoretical analyses for this purpose. In this paper, an experimental study has been presented to verify that the propagating pressure is affected by the resonances of the piping structure in addition to the effect of the fluid on the system damping. The case of pressure variations in the fluid contained in a piping system with no flow velocity has also been considered here.

A simple plane piping system, constructed from PVC pipes, was used in this study and is shown in Fig. 1. The numerical data of the piping structure used are: pipe outer diameter, $D=22 \mathrm{~mm}$, inner diameter, $d=15 \mathrm{~mm}$ and each pipe length, $L=980 \mathrm{~mm}$. The system was clamped at one end to a rigid support and the other end remained free.

Since the dynamic modulus of elasticity and the density of the pipe material were not known, preliminary tests were carried out to obtain these quantities. The main experiment was conducted to obtain the resonance frequencies of the piping system from a set of frequency response functions (FRF) of the piping structure, in the transverse mode, and using fluid pressure measurements. The damping ratio associated with each peak was assumed to be the modal damping ratio. The modal damping ratios corresponding to each resonance frequency were calculated from the FRF for the empty piping system and that fully-water-filled one using the onehalf power point bandwidth technique. The experimental results for the resonance frequencies were compared with the theoretical ones that were obtained in reference $e^{4}$ by use of the finite element technique. The experimental results were found to be in good agreement with the theoretical ones. The calculated modal damping ratios of the empty piping system were compared with those of the fully-filled piping system to illustrate the effect of the fluid on the system damping.

\section{INSTRUMENTATION SET-UP}

The required frequency response measurements were carried out using a Dual Channel Signal Analyser, type B\&K 2034. The frequency response functions were obtained by exciting the system with a measurable force, and by measuring the associated response. Then these results were transformed into the frequency domain using the Fast Fourier Transform analyser and the ratio between the two spectra was calculated. The peaks of the measured frequency response functions correspond to the system resonances. An illustrative layout for the instrumentation set up is shown in Fig. 2.

The excitation force was obtained from an impact hammer applied in the transverse direction. This force was measured by a piezoelectric force transducer and the resulting signal was applied to the channel " $A$ " input of the analyser (ref- 